\title{
Robotic Mobile System's Performance-Based MIMO-OFDM Technology
}

\author{
Omar Daoud ${ }^{1}$ and Omar Alani ${ }^{2}$ \\ doi:10.3991/ijim.v3s2.923 \\ ${ }^{1}$ Philadelphia University, Amman, Jordan \\ ${ }^{2}$ University of Leeds, Leeds, United Kingdom
}

\begin{abstract}
In this paper, a predistortion neural network (PDNN) architecture has been imposed to the Sniffer Mobile Robot (SNFRbot) that is based on spatial multiplexed wireless Orthogonal Frequency Division Multiplexing (OFDM) transmission technology. This proposal is used to improve system performance by combating one of the main drawbacks that is encountered by OFDM technology; Peakto-Average Power Ratio (PAPR). Simulation results show that using PDNN resulted in better PAPR performance than the previously published work that is based on linear coding, such as Low Density Parity Check (LDPC) codes and turbo encoding whether using flat fading channel or a Doppler spread channel.
\end{abstract}

Index Terms-Neural Network, LDPC codes, Robot, MIMO, and OFDM.

\section{INTRODUCTION}

Researchers recently began to pay more attention to ubiquitous robots $[1,2]$, due to the rapid growth of wireless communication services. The previously proposed Sniffer Mobile Robot (SNFRbot) [3] requires a specific combination of wireless communication, user interface and signal processing techniques to assist humans in everyday tasks. SNFRbot is a smart robot that monitors dangerous areas and supplies the administrator with vital information and, in some cases, plays the coadministrator role. This monitoring is accomplished by sending live videos through Multi-Input Multi-Output Orthogonal Frequency Division Multiplexing (MIMOOFDM) modems to the administrator. The administrator should notify the SNFRbot that the situation on hand is under control; otherwise it will act as a co-administrator to give temporary solutions.

OFDM technology is based on the advantages offered by the Fast Fourier Transform (FFT) algorithm. These advantages include reduction in the size of circuits, ability to vary transmission capacity by changing the number of subcarriers and tackling the effect of Inter-Symbol Interference (ISI) [4-6]. Moreover, at any wireless link, employing multiple antennas at both ends has recently been shown to have the probability of achieving extraordinary data rates, even in a rich scattering environment $[7,8]$. Such technology is known as spatial multiplexing or BLAST and allows an increase in bit rate in a wireless radio link without additional power or bandwidth consumption [9]. In a time-varying frequencyselective fading channel, the BLAST system may suffer from severe performance degradation. The combination of these two powerful technologies; MIMO and OFDM, will prevent this degradation. It has been shown [7,9], however, that OFDM suffers from high sensitivity to nonlinear distortion caused by components in the transmitter part, such as Digital to Analogue Converters (DAC), mixers and High Power Amplifiers (HPA). Thus, the output signal will suffer from intermodulation distortion resulting in energy being generated at frequencies outside the allocated bandwidth. To this end, Daoud et al. [10, 11], introduced a novel technique to improve the OFDM systems' efficiency by alleviating the PAPR problem based on linear coding techniques.

In this work, we present an improvement to the SNFRbot-based coded MIMO-OFDM system proposed in [3] by combating the PAPR problem and its effect on the bit-error-rate (BER) and signal-to-noise ratio (SNR). For this purpose, Neural Network (NN) architecture is built to be compared with the achieved efficiency, which was based on linear coding technique previously proposed in the literature. A detailed description of the SNFRbotbased MIMO-OFDM systems' architecture is given in Section II. To verify the efficiency of the proposed technique, hardware and computer simulation results are given in Section III, followed by the conclusion in Section IV.

\section{SNFRBOT-BASED MIMO-OFDM SYSTEMS' ARCHITECTURE}

The general structure of the proposed work is shown in Fig. 1. It consists of four layers; the sensor/video layer, the software/hardware (SW/HW) layer, the wireless layer and the administrator layer. In the sensor/video layer, the SNFRbot monitors the environment's safety. If any sudden accident happens that causes a high deadly gas concentration or a high temperature in the surroundings, the video cam mode will be activated to send live video to the administrator. In the second layer, both of the SNFRbot hardware and software are reconfigurable to satisfy the environment conditions, such as the SNFRbot speed. The third layer allows the SNFRbot to send the live videos through a coded MIMO-OFDM modem. This modem consists of four parts as shown in Fig. 1. The receiver stage is the inverse process of the MIMO-OFDM transmitter block.

For the coded MIMO-OFDM block, the turbo encoder of $1 / 2$ coding ratio is used for the coding stage. Two different modulation techniques are used; QPSK and 16QAM, together with a Vertical Bell Laboratories Layered Space-Time (V-BLAST) MIMO encoder. VBLAST is used for increasing the overall throughput expressed in terms of bits/symbol for OFDM symbols, 


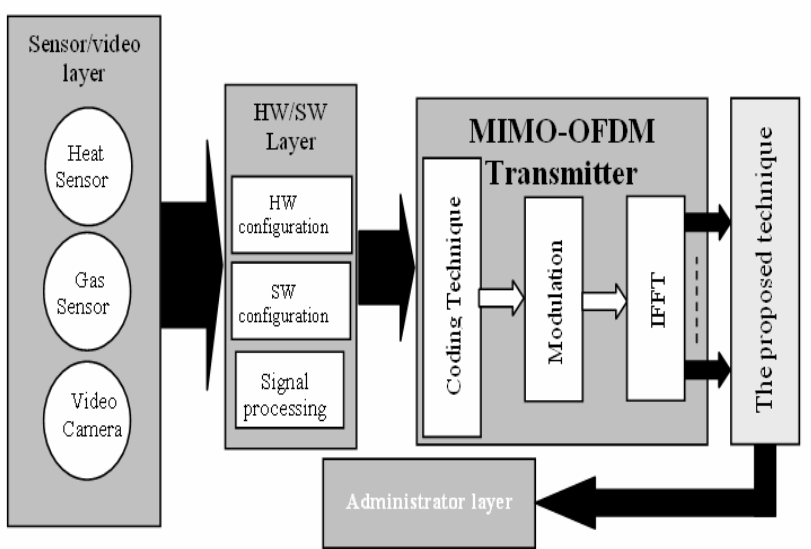

Figure 1. Generic structure of SNFRbot-based MIMO-OFDM systems' architecture

generated by applying the IFFT. After the IFFT stage and due to the coherent addition of the independently modulated subcarriers to produce OFDM symbol, a large PAPR ratio could appear. Thus we try to reduce the effect of these peaks by applying the PDNN and then compare it to the achieved results from our previously published work of using linear coding techniques [11,16,17]. Subsection A describes the MIMO-OFDM-based PDNN systems, while Subsection B gives a brief overview of the previously published work to combat the PAPR problem. In OFDM configuration, the transmitted signal from one antenna is expressed as in [7]:

$s(t)=\sum_{k=-\infty}^{\infty} \sum_{i=0}^{N-1} d_{i}(k) \exp \left(j 2 \pi f_{i}\left(t-k T_{s}\right)\right) f\left(t-k T_{s}\right)$

where, $d_{i}$ is a complex input symbol after the modulation stage, $f_{i}$ is the frequency of the $i$-th subcarrier, $T_{s}$ is the OFDM symbol duration.

\section{A. Combating the PAPR-based NN}

The use of $N$-points Inverse FFT (IFFT) in the OFDM transmitter causes the PAPR problem. This arises when $N$ signals are added with the same phase in IFFT stage to produce OFDM symbol. A high peak power equals to $N$ times the average power occasionally appears as a result of this addition process. Thus, the PAPR definition can be written as:

$$
P A P R=10 \times \log _{10}\left\{\frac{P_{\text {peak }}}{P_{\text {avg }}}\right\}
$$

where, $P_{\text {peak }}$ is the maximum power of an OFDM symbol, and $P_{\text {avg }}$ is the average power. The PAPR can be reformulated as:

$$
P A P R=\frac{|x(t)|^{2}}{\frac{1}{N T_{s}} \int_{0}^{N T_{s}}|x(t)|^{2} d t}
$$

where $x(t)$ is the OFDM symbol at time $t$. Moreover, the average power of the OFDM symbol in (2) can be written as:

$$
\begin{gathered}
P_{\text {avg }}=\sum\left\{\left|x_{i}(t)\right|^{2}\right\}=\frac{1}{T_{s}} \int_{0}^{T_{s}}|x(t)|^{2} d t \\
P_{a v g}=\frac{1}{T_{s}} \int_{0}^{T_{s}}\left|\sum_{v=0}^{N-1} c_{v} e^{j 2 \pi \frac{v}{N T} T_{s}}\right|_{v}^{2} d t \\
=\frac{1}{T_{s}} \int_{0}^{T_{s}}\left(\sum_{v=0}^{N-1} c_{v} \cos \left(2 \pi \frac{v}{N T_{s}} t_{v}\right)\right)^{2}+\left(\sum_{v=0}^{N-1} c_{v} \sin \left(2 \pi \frac{v}{N T_{s}} t_{v}\right)\right)^{2} d t
\end{gathered}
$$

$$
P_{a v g}=\frac{1}{T_{s}} \int_{0}^{T_{s}}\left(\sum_{v=0}^{N-1} c_{v}^{2}\right) d t
$$

where $c_{v}$ is the magnitude of the modulated data. For simplicity, if BPSK modulation is used without any channel coding techniques, then $\left|c_{v}\right|=1$, when $\mathrm{t} \in[0, \mathrm{~T}]$. This can be substituted in (6). The result from this substitution leads to a direct relationship between the average power and the total number of the IFFT points $N$, which can be taken directly from

$$
P_{a v g}=N \times \frac{1}{T s} \int_{0}^{T_{s}} c_{v}^{2} d t=N
$$

From (7), the average power is equal to the number of the BPSK-modulated orthogonal subcarriers. As already mentioned, the maximum peak amplitude is $N$, therefore, the maximum power of the OFDM symbol is $N^{2}$. As a result, from (2), the PAPR of uncoded BPSK will be $10 \times \log _{10}\left(P_{a v g}\right) \mathrm{dB}$. Therefore, the PAPR will decrease if the average power of the OFDM symbol is decreased. This mathematical derivation was the basis of our previously published work to combat the effect of the PAPR drawback on the MIMO-OFDM systems $[11,16,17]$.

One of the affected components by the nonlinearity problem in MIMO-OFDM systems is the Radio Frequency Power Amplifier (RFPA). Imposing the PDNN can improve MIMO-OFDM systems performance; as it is the simplest way of linearization for RFPA. This is due to the PDNN ability of simultaneous BW linearization [9]. Fig. 2 shows the structure of the PDNN, which is applied to the proposed technique block in Fig. 1. Here, the generated OFDM signal is fed into a NN before it goes through the RFPA. The NN weight should be trained to give the best output according to the RFPA, which is also attained by the learning algorithm [13].

The setup process of any NN is based on adjusting the weights matrix $(W)$, which reduces the error between the actual output and the desired output. In order to adjust $W$, the back propagation algorithm is used to determine how the error changes as each weight is modified (slightly incremented or decremented), i.e. it is used to calculate the error derivatives of $W ; E(W)$. 


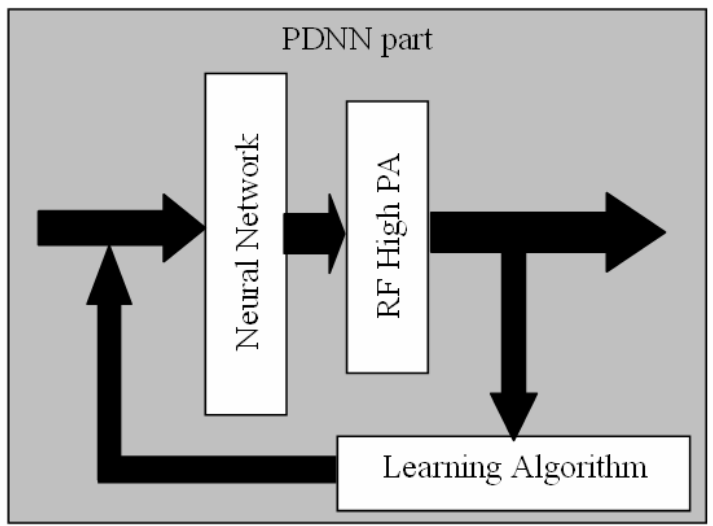

Figure 2. PDNN part structure

In this work, the PDNN will be trained on the previously published results of combating the PAPR based on linear coding techniques as in $[7,14,15]$. In the back propagation algorithm, the weights of the layers will be adjusted in the steepest descent algorithm; the negative of the gradient. However, the conjugate gradient algorithm is favored to be used as it achieves the fastest convergence $[9,11]$.

For any weight vector in the weight space, $W_{o}$, the second order Taylor series approximation is given as [9]:

$$
E(W)=E\left(W_{o}\right)+g^{T} \Delta W+\frac{1}{2}(\Delta W)^{T} H \Delta W
$$

where $g=\left.\frac{\partial E}{\partial W}\right|_{W=W_{o}}$ and $H=\left.\frac{\partial^{2} E}{\partial W^{2}}\right|_{W=W_{o}}$ are the gradient vector and the Hessian matrix, respectively. Taking the gradient of $E(W)$ and equating it with zero (i.e. $\frac{\partial E}{\partial W}=g+H \Delta W=0$ ) will lead to the minimum of the function in (8) and then the optimal value for $W$ is $W_{o}-H^{-1} g$, which is the gradient algorithm basic formulation. Here, the main concern is to compute $H^{1}$. Thus the conjugate gradient algorithm could be used with its basic updating equation as follow:

$$
W(k+1)=W(k)+\eta(k) \Delta W(k)
$$

where $\eta(k)$ and $\Delta W(k)$ are the time varying learning parameter and the incremental weight vector, respectively. For the incremental weight vector, to satisfy the conjugate condition between two successive vectors an updating parameter should be defined. This parameter has some expressions in the literature, such as Polak-Ribier formula, Fletcher-Reevers formula and Powell-Beale restarts $[12,15]$. In this work, the Powell-Beale algorithm will be used since it improves the training efficiency by choosing the best reset point in the search direction (in all conjugate gradient algorithms, the search direction will be reset to the negative of the gradient periodically).

\section{B. Combating the PAPR-based Linear Coding}

Based on the mathematical derivations in (2)-(7), a novel model to reduce the appearance of large power peaks in the OFDM symbols was presented in [10]. Fig. 3 is a flowchart to describe the main stages of the proposed technique.

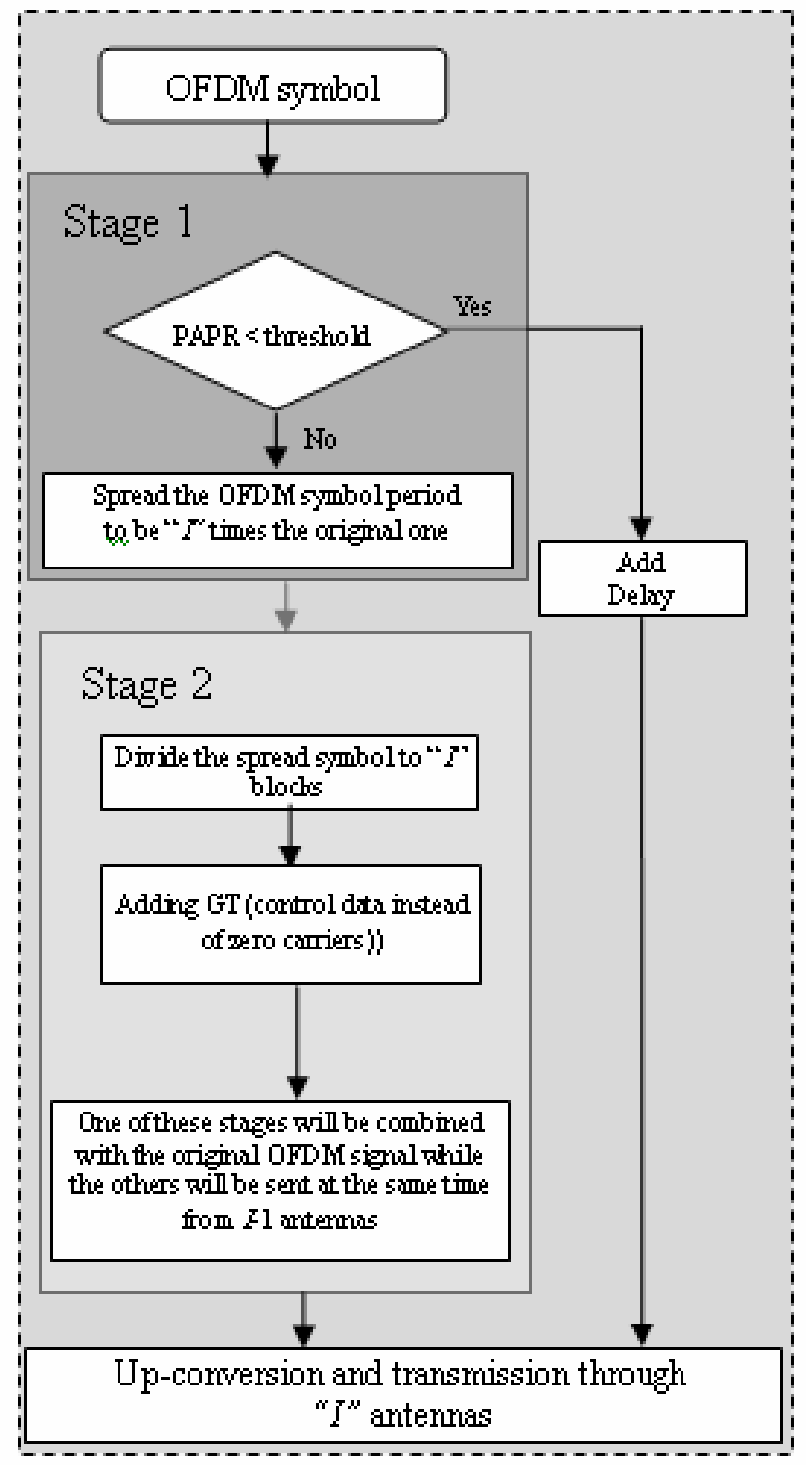

Figure 3. The flowchart of the proposed technique

In the first stage, the proposed technique starts with a check on the OFDM symbol to see if it is suffering from high PAPR values or not (this depends on the application itself; in our case the threshold is settled to be $10 \mathrm{~dB}$ based on mobile communication systems [10]). If the PAPR is within a set tolerance, " $I$ " coded symbols generated through a Space Time Block Coder (STBC) will be transmitted through " $I$ " number of antennae. If the PAPR, however, exceeds a predetermined acceptable threshold, the symbol will pass through linear coding block having first removed the guard interval.

In $[10,16,17]$, different linear coding techniques have been used to accomplish the aim of reducing PAPR, such as convolutional encoding, LDPC codes and turbo coding. The period of the affected symbol will be spread according to the chosen spreading rate, to be " $I$ " times the original one, where " $I$ " is the spreading rate.

The second stage of the proposed technique deals with the new symbol and choosing the data with the lowest PAPR value. The spread symbol will be mapped to " $I$ " parallel blocks, each of which has the same duration as the conventional symbol duration. The number of iterations that is needed to calculate the PAPR of each block, and 
choosing the lowest value is given by $[((I-1) N)+1]$. Thus, the proposed technique will add a first order complexity in according to the variable $N$. Due to the achieved spreading by using linear coding; these blocks will have a PAPR value less than the threshold value. This will be the starting point in our work. We have got the affected OFDM symbols and the desired ones that were achieved from the previously proposed work; these are the needed data to train the PDNN in this work. This will simplify the previously published work since we get used to use a complicated receiver based on [18] to ensure the transmission of the processed OFDM symbol simultaneously with the OFDM signal from $I$ antennas.

The SNFRbot prototype is approximately in the size of $6 \times 10^{-3} \mathrm{~m}^{3}$ as shown in Fig. 4. At this stage, it has the following parts; motor drive; a MIMO-OFDM modem designed using field-programmable gate array (FPGA); a microcontroller; a power supply; sensors and a video camera.

In this work, the coded MIMO-OFDM modem has been built in accordance to the IEE $802.11 \mathrm{~g} / \mathrm{h}$ specifications to test the wireless communication of SNFRbot. The algorithm was implemented in FPGA available from Xilinx [19]. The algorithm was decomposed into its functional blocks and these blocks were implemented using Verilog, a well known hardware description language (HDL). 'ISE' [20], a development environment provided by Xilinx, incorporates a Verilog synthesis tool chain allowing the high-level description to be compiled and optimized. Elements of this tool chain allow the generation of a bit stream file from which the FPGA devices can be directly programmed. Furthermore, the ISE environment also provides a behavioral simulator that allows the performance of the design to be examined postsynthesis, i.e. complete with all of the routing delays and imperfections that would be present in the physical instantiation of the design in an actual FPGA. This proved to be an extremely useful tool during the implementation of this technique.

The blocks described in Fig.1 were successively implemented in Verilog HDL, each being tested and evaluated using the behavioral simulator before the next block in the chain was implemented. Additional blocks were also implemented to provide the necessary timing, synchronization and handshake signals necessary to ensure correct data transport between the blocks. During the compilation and synthesis process, the ISE tool chain generates a report showing device utilisation. Once the system was implemented in Verilog HDL, this report was used to determine the overall size and structure of the FPGA device required to implement this technique.

The full implementation was found to fit comfortably in a Xilinx XC2S150 part; one of the Spartan 2E series. The utilisation report indicated that approximately $85 \%$ of the device was used. The Spartan 2E series is a mature, readily available low cost family, showing clearly that the technique can be implemented using low-cost hardware.

It is possible to reduce device utilisation even further. In the implementation used during this research, the IFFT block was implemented directly from the flow graph to expedite the development.

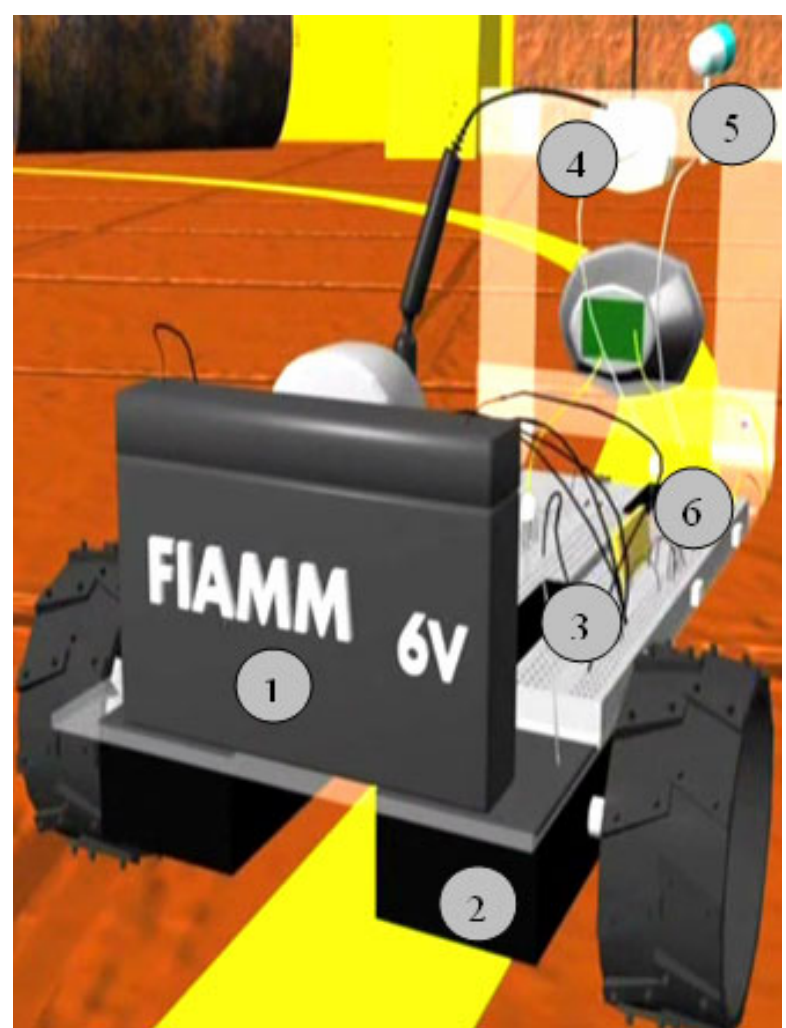

Figure 4. The prototype of SNFRbot: 1) 6V battery, 2) motor driver, 3) microcontroller, 4) video cam, 5) heat sensor, and 6) gas sensor.

\section{SNFRBOT-BASED MIMO-OFDM SYSTEMS' ARCHITECTURE}

Experiments have been carried out to evaluate the MIMO-OFDM based PDNN performance. The simulation environment consists of the following; uniformly distributed randomly generated data sequence, channel coding rate equals to $1 / 2$, different modulation techniques (BPSK and 16QAM), IFFT size of 1024. For spreading purposes, there are three different linear coding techniques which have been used with spreading rate, $I=3$; a memory-2 turbo coding with generator polynomial $g=[1$

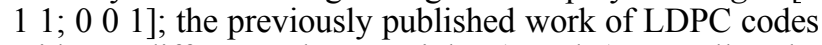
with two different column weights (6 and 7), as well as the following values: $m=64, n=512, b=64, \gamma=3$ and $d_{\text {min }}=$ 8 ; and memory-2 convolutional coding. For the PDNN, two learning parameter values were used $\eta=0.1$ and 0.01 and the learning method used was Powell-Beale gradient back-propagation.

Fig. 5 shows the cumulative complementary density function (CCDF) plot of applying the linear coding techniques to overcome the PAPR problem in the MIMOOFDM system. The CCDF plots for the PAPR reduction performance with BPSK and 16QAM modulation techniques are depicted in Fig. 5.a and Fig. 5.b respectively. These figures compare the threshold value against the probability of the PAPR exceeding that threshold value. From these figures the improvements are shown over what have been achieved in our previous published work in $[16,17]$. 


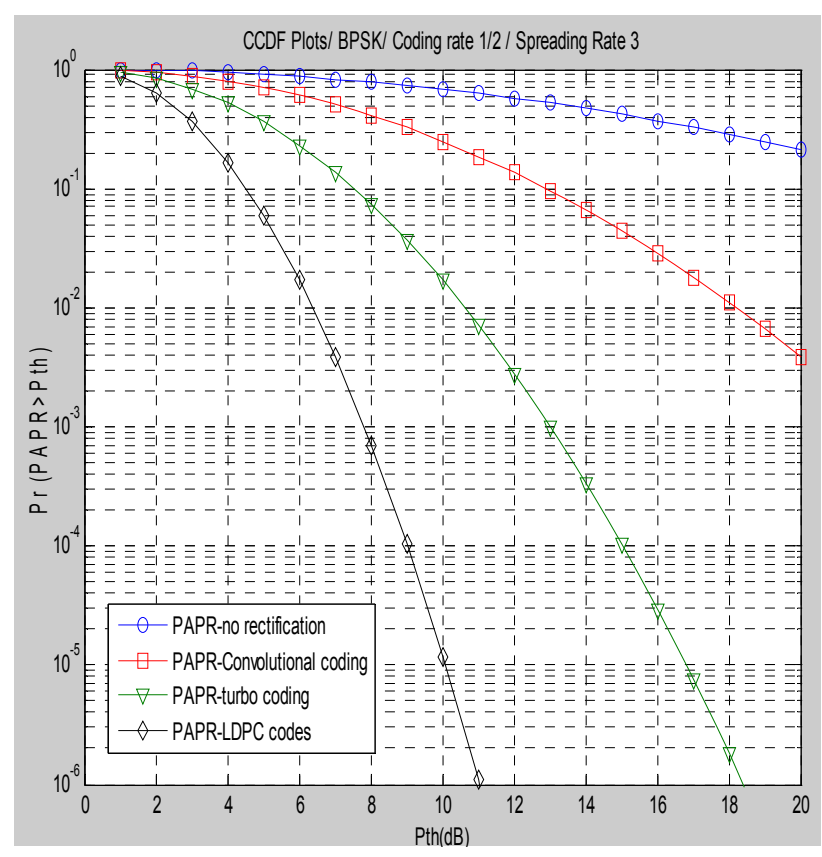

(a)

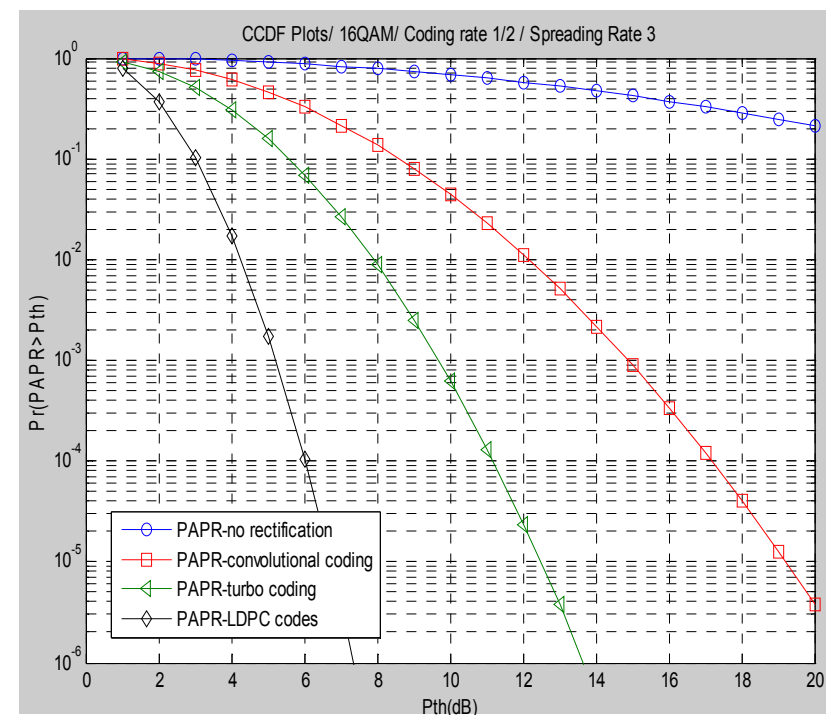

(b)

Figure 5. Probabilities of PAPR exceeding a certain threshold, $\mathrm{P}_{\text {th }}$, when applying linear codes (for BPSK and 16QAM modulation process, coding rate equals to $1 / 2$, and spreading rates 3 in figure 5 .a and 5.b, respectively).

After the PAPR reduction is complete based on different linear coding techniques, both of the affected OFDM symbol and the chosen one after applying the spreading techniques have been used in the learning process of the PDNN. The overall MIMO-OFDM system based PDNN has been modelled through a multipath fading channel. Table I shows the achieved results of the training procedure.

TABLE I.

POWELL-BEALE CONJUGATE GRADIENT BACK-PROPAGATION

\begin{tabular}{|c|c|c|}
\hline $\begin{array}{c}\text { Learning } \\
\text { parameter } \boldsymbol{\eta}\end{array}$ & MSE & $\begin{array}{c}\text { Number of trained } \\
\text { OFDM symbols }\end{array}$ \\
\hline 0.1 & $4.34 \times 10^{-6}$ & 1000 \\
\hline 0.01 & $5.151 \times 10^{-6}$ & 1000 \\
\hline
\end{tabular}

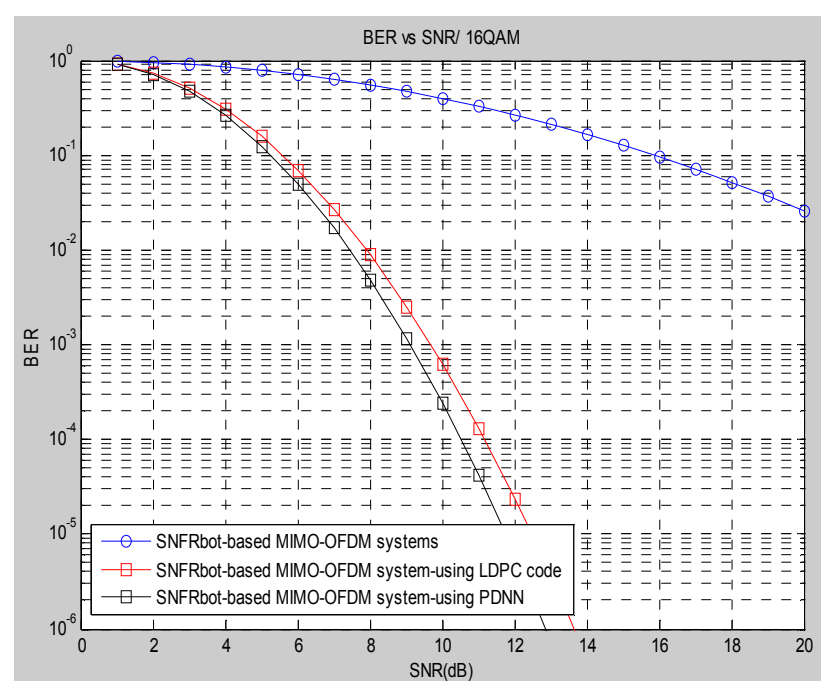

Figure 6. BER vs. SNR comparison between the achieved results of using linear coding techniques and the ones of PDNN through a multipath fading channel.

From Fig. 5.a, it can be seen that at a PAPR threshold of $10 \mathrm{~dB}$, the probability of the PAPR values that exceed this threshold is reduced from $0.79 \times 10^{-1}$ to $1.095 \times 10^{-5}$ using LDPC codes which is the best results achieved over the use of both of turbo coding and convolutional encoding, while Fig. 5.b shows that using 64QAM instead BPSK enhances the PAPR reduction ratios. From Fig. 6, using the PDNN slightly enhances the BER vs. SNR plots. As an example at $10 \mathrm{~dB}$ SNR the BER has been enhanced from $6.5 \times 10^{-4}$ to $2.1 \times 10^{-4}$.

\section{CONCLUSION}

In this paper, a work based on neural networks has been introduced to enhance the SNFRbot based OFDM systems performance. Basically, the learning process of the proposed NN is based on the previously published work.

The simulation results demonstrate the reduction of PAPR for different linear coding techniques, and show that the use of LDPC gives the best result of reduction. Also, increasing the weight of modulation techniques has improved the achieved results. From the obtained results, imposing $\mathrm{NN}$ instead of using linear coding gives a slight improvement in the PAPR reduction values.

In this work, using the $\mathrm{NN}$ reduces the added complexity at the receiver side since there is no need to send a control data as was the case in previously published work. The use of MIMO technology in this work is to improve the system capacity by using the Vertical-BLAST (V-BLAST) technology.

\section{REFERENCES}

[1] J. Kim, K. Lee, Y. Kim, N. Kuppuswamy and J. Jo, "Ubiquitous Robot: A New Paradigm for Integrated Services", IEEE International Conference on Robotics and Automation, Rome, Italy, pp. 2853-2858, Apr. 2007.

[2] D. Fox, W. Burgard, H. Kruppa, and S. Thrun, "A probabilistic approach to collaborative multi-robot localization", Autonomous Robots, vol. 8, no. 3, pp. 325-344, Jun. 2000. (doi:10.1023/A:1008937911390)

[3] O. Daoud and O. Alani, "MIMO-OFDM system performance using LDPC code for a Mobile Robot", The First International ICST Conference on Communications Infrastructure, Systems and Applications in Europe, London, (Accepted), 11-13 Aug. 2009. 
[4] J. A. C. Bingham, "Multicarrier modulation for data transmission: An idea whose time has come," IEEE Communication Magazine, vol. 28, no. 5, pp. 5-14, May 1990. (doi:10.1109/35.54342)

[5] S. Hara, M. Mouri, M. Okada and N. Morinaga, "Transmission performance analysis of Multi-Carrier Modulation in frequency selective fast Rayleigh fading channel," Wireless Personal Communications, vol. 2, no. 4, pp. 335-356, Jan.-Feb. 1996. (doi:10.1007/BF01099340)

[6] T. M. Schmidl and D. C. Cox, "Blind synchronization for OFDM," Electronics Letters, vol. 33, no. 2, pp. 113-114, Jan. 1997. (doi:10.1049/el:19970089)

[7] R. v. Nee and R. Prasad, "OFDM for wireless multimedia communications", Artech House Inc. Publishing-Norwood (MAUSA), 2000.

[8] M. Juntti, M. Vehkapera, J. Leinonen, V. Zexian, D. Tujkovic, S. Tsumura and S. Hara, "MIMO MC-CDMA Communications for Future Cellular Systems", IEEE Communication Magazine, vol. 43, no. 2, pp. 118-124, Feb. 2005. (doi:10.1109/MCOM. 2005.1391510)

[9] S. Han and J. Lee, PAPR Reduction of OFDM Signals Using a Reduced Complexity PTS Technique, in IEEE Signal Processing Letters, vol. 11, no. 11, pp. 887-890, Nov. 2004.

[10] M. Al-Akaidi and O. Daoud, "Reducing the Peak-to-Average Power Ratio Using Turbo Coding", IEE Proceeding Communications, vol. 153, no. 6, pp. 818-821, Dec. 2006. (doi:10.1049/ip-com:20060061)

[11] M. Al-Akaidi, O. Daoud and S. Linfoot, "A new Turbo Coding Approach to reduce the Peak-to-Average Power Ratio of a MultiAntenna-OFDM", International Journal of Mobile Communications, vol. 5, no. 3, pp. 357-369, 2007. (doi:10.1504/ IJMC.2007.012399)

[12] M. Gupta, L. Jin and N. Homma, "Static and Dynamic Neural Networks", John Wiley and Sons. Inc. 2003. (doi:10.1002/0471427950)

[13] H. H. Wang, H. Zhang, W. Ye and S. L. Feng, "Neural network predistortion based on error-filtering algorithm in OFDM system", Proceedings of the International Conference on Wireless Communications, Networking and Mobile Computing (WCNC), vol. 1, pp. 193- 196, Sep. 2005. (doi:10.1109/WCNM. 2005.1544015)

[14] V. B. Rao, H. V. Rao, "C++ Neural Networks and Fuzzy Logic", MIS: Press, 1995

[15] "Matlab: Neural Networks Toolbox User's Guide", pdf help format, The Mathworks Inc. Jul. 2002.
[16] M. Al-Akaidi, O. Daoud and J. Gow, "MIMO-OFDM-based DVB-H systems: A Hardware design for a PAPR reducing technique", IEEE Transaction on Consumer Electronics, vol. 52, issue 4, pp. 1201-1206, Nov. 2006. (doi:10.1109/TCE.2006.273134)

[17] O. Daoud and O. Alani, "Reducing PAPR by Utilization of LDPC CODE ", IET Communication Proceedings, vol. 3, no. 4, pp. 520529, Apr. 2009. (doi:10.1049/iet-com.2008.0344)

[18] M. Muck, M. de Courville and P. Duhamel "A Pseudorandom Postfix OFDM Modulator-Semi-blind Channel Estimation and Equalization," IEEE Transactions on Signal Processing, vol. 54, no. 3, pp. 1005- 1017, Mar. 2006. (doi:10.1109/TSP.2005.863012)

[19] Xilinx- manufacturer of FPGA silicon and development tools, http://www.xilinx.com. Last accessed 11/03/2009.

[20] Xilinx:ISE, http://www.xilinx.com/ise/logic design prod/web pack.htm. Last accessed 11/03/2009.

\section{AUTHORS}

O. R. Daoud, has $\mathrm{PhD}$ in Communication and Electronics Engineering from DMU/ UK 2006. He joined Philadelphia University in 2007 as Assistant Professor. His current work is about achieving the Quality of Service for the 4th Generation of the Wireless and Mobile Communication Systems by combining the advantages of the OFDM and the multiple antenna technology. (odaoud@philadelphia.edu.jo)

O.Y. Alani, has $\mathrm{PhD}$. degree in Telecommunication Engineering from De Montfort University, UK in 2005. He worked as a lecturer of communication systems at DMU. After that he joined the Institute of Advanced Telecommunications, University of Wales Swansea in the Networks group. His research interests include Radio resource management and location/mobility management in next generation mobile communication systems, diversity and adaptive modulation techniques as well as Ad hoc networks. He is currently with the School of Electronic \& Electrical engineering, University of Leeds as research fellow in the wireless communication field. (o.y.k.alani@leeds.ac.uk)

Submitted 4 May 2009. Published as resubmitted by the authors on 9 October 2009. 